\title{
Corrigendum: On an Enriques surface associated with a quartic Hessian surface
}

http://dx.doi.org/10.4153/CJM-2018-022-7

\section{Ichiro Shimada}

In the original article [1], we made a mistake in the calculation of the number of $\operatorname{Aut}(Y)$-equivalence classes of RDP-configurations on an Enriques surface $Y$ covered by a $K 3$ surface birational to a general quartic Hessian surface. Theorem 1.5 and Table 1.2 of the paper should be replaced by the following:

Theorem 1.5 Up to the action of $\operatorname{Aut}(Y)$, the Enriques surface $Y$ has exactly 33 nonempty RDP-configurations. Their ADE-types are given in Table 1.2.

\begin{tabular}{|c|c|c|c|}
\hline$A D E$-type & Number & $A D E$-type & Number \\
\hline$E_{6}$ & 1 & $A_{3}+A_{1}$ & 1 \\
\hline$A_{5}+A_{1}$ & 5 & $2 A_{2}$ & 1 \\
\hline $3 A_{2}$ & 1 & $A_{2}+2 A_{1}$ & 1 \\
\hline$D_{5}$ & 1 & $4 A_{1}$ & 5 \\
\hline$A_{5}$ & 1 & $A_{3}$ & 1 \\
\hline$A_{4}+A_{1}$ & 1 & $A_{2}+A_{1}$ & 1 \\
\hline$A_{3}+2 A_{1}$ & 5 & $3 A_{1}$ & 2 \\
\hline $2 A_{2}+A_{1}$ & 1 & $A_{2}$ & 1 \\
\hline$D_{4}$ & 1 & $2 A_{1}$ & 1 \\
\hline$A_{4}$ & 1 & $A_{1}$ & 1 \\
\hline
\end{tabular}

Table 1.2: RDP-configurations on $Y$.

Received by the editors September 17, 2020.

Published online on Cambridge Core December 10, 2020.

AMS subject classification: $14 \mathrm{~J} 28$.

Keyword: Enriques surface. 
The corrected version of the theorem gives us the following:

Corollary The automorphism group $\operatorname{Aut}(Y)$ acts on the set of smooth rational curves on Y transitively.

The mistake in the original proof is located in the second paragraph of Section 7.6. Let $\mathcal{F}$ be the set of maximal nonideal faces of $D_{Y}$, and we consider the mapping $F \mapsto$ $\mathcal{R}(F)$ on $\mathcal{F}$. Even when two faces $F \in \mathcal{F}$ and $F^{\prime} \in \mathcal{F}$ are not aut $(Y)$-equivalent, the RDP-configurations $\mathcal{R}(F)$ and $\mathcal{R}\left(F^{\prime}\right)$ can be in the same aut $(Y)$-orbit. This happens when $F^{g}$ and $F^{\prime}$ span a same linear subspace in $S_{Y} \otimes \mathbb{R}$ for some automorphism $g \in$ $\operatorname{aut}(Y)$.

We briefly explain the algorithm to obtain the correct version of Theorem 1.6. The detail of this algorithm will be explained in the forthcoming paper by the author in a more general setting.

Let $F$ be an element of $\mathcal{F}$, and we put $\Gamma:=\mathcal{R}(F)$. Let $\langle\Gamma\rangle$ be the sublattice of $S_{Y}$ generated by $\Gamma$, and $\langle\Gamma\rangle^{\perp}$ the orthogonal complement of $\langle\Gamma\rangle$ in $S_{Y}$. Then $\langle\Gamma\rangle^{\perp}$ is a hyperbolic lattice of rank $10-\mu$, where $\mu:=|\Gamma|$ is the total Milnor number of the surface $\bar{Y}$ corresponding to $F$. We consider the positive cone

$$
\mathcal{P}_{\langle\Gamma\rangle^{\perp}}:=\mathcal{P}_{Y} \cap\left(\langle\Gamma\rangle^{\perp} \otimes \mathbb{R}\right)
$$

of $\langle\Gamma\rangle^{\perp}$. A $\langle\Gamma\rangle^{\perp}$-induced chamber is a closed subset of the cone $\mathcal{P}_{\langle\Gamma\rangle^{\perp}}$ of the form $\mathcal{P}_{\langle\Gamma\rangle^{\perp}} \cap D$, where $D$ is an induced chamber in $\mathcal{P}_{Y}$ such that $\mathcal{P}_{\langle\Gamma\rangle^{\perp}} \cap D$ contains a nonempty open subset of $\mathcal{P}_{\langle\Gamma\rangle^{\perp}}$. For example, the face $F$ is a $\langle\Gamma\rangle^{\perp}$-induced chamber

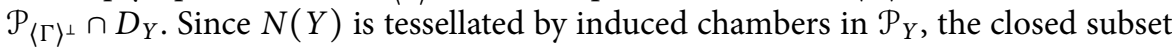
$\mathcal{P}_{\langle\Gamma\rangle^{\perp}} \cap N(Y)$ of $\mathcal{P}_{\langle\Gamma\rangle^{\perp}}$ is tessellated by $\langle\Gamma\rangle^{\perp}$-induced chambers. We denote by $V_{\Gamma}$ the set of $\langle\Gamma\rangle^{\perp}$-induced chambers contained in $\mathcal{P}\langle\Gamma\rangle^{\perp} \cap N(Y)$. Then, the group

$$
\operatorname{aut}(Y, \Gamma):=\left\{g \in \operatorname{aut}(Y) \mid \Gamma^{g}=\Gamma\right\}
$$

acts on $V_{\Gamma}$.

Let $\mathcal{P}_{\langle\Gamma\rangle^{\perp}} \cap D_{0}$ be an element of $V_{\Gamma}$, where $D_{0}$ is an induced chamber contained in $N(Y)$. Then,

- we can make the list of all induced chambers $D_{0}^{\prime}$ contained in $N(Y)$ such that $\mathcal{P}_{\langle\Gamma\rangle^{\perp}} \cap D_{0}=\mathcal{P}_{\langle\Gamma\rangle^{\perp}} \cap D_{0}^{\prime}$,

- we can make the list of all $\langle\Gamma\rangle^{\perp}$-induced chambers $\mathcal{P}_{\langle\Gamma\rangle^{\perp}} \cap D^{\prime}$ adjacent to $\mathcal{P}_{\langle\Gamma\rangle^{\perp}} \cap$ $D_{0}$,

- for another $\langle\Gamma\rangle^{\perp}$-induced chamber $\mathcal{P}_{\langle\Gamma\rangle^{\perp}} \cap D_{1}$, where $D_{1}$ is an induced chamber contained in $N(Y)$, we can enumerate all the elements $g$ of aut $(Y)$ that maps $D_{0}$ to $D_{1}$, and

- combining the algorithms above, we can make the list of all $g \in \operatorname{aut}(Y)$ that maps $\mathcal{P}_{\langle\Gamma\rangle^{\perp}} \cap D_{0}$ to $\mathcal{P}_{\langle\Gamma\rangle^{\perp}} \cap D_{1}$.

Then, we can make a complete set of representatives

$$
\mathcal{P}_{\langle\Gamma\rangle^{\perp}} \cap D^{(0)}, \ldots, \mathcal{P}_{\langle\Gamma\rangle^{\perp}} \cap D^{(m)}
$$

of the orbits of the action of $\operatorname{aut}(Y, \Gamma)$ on $V_{\Gamma}$, where $D^{(0)}, \ldots, D^{(m)}$ are induced chambers contained in $N(Y)$. 
Let $F^{\prime}$ be another element of $\mathcal{F}$ such that $\Gamma^{\prime}:=\mathcal{R}\left(F^{\prime}\right)$ has the same $A D E$-type as $\Gamma$. Then $\Gamma^{\prime}$ belongs to the same aut $(Y)$-orbit as $\Gamma$ if and only if there exist a $\langle\Gamma\rangle^{\perp}$-induced chamber $\mathcal{P}_{\langle\Gamma\rangle^{\perp}} \cap D^{(k)}$ in the representatives (1.1) and an element $g$ of aut $(Y)$ that maps $F^{\prime}$ to $\mathcal{P}\langle\Gamma\rangle^{\perp} \cap D^{(k)}$. Applying this criterion to all pairs of faces in $\mathcal{F}$, we obtain the orbit decomposition by aut $(Y)$ of RDP-configurations of smooth rational curves on $Y$.

We apologize for possible confusion that this mistake may have caused.

\section{Reference}

[1] I. Shimada, On an Enriques surface associated with a quartic Hessian surface. Canad. J. Math. 71(2019), 231-246.

Department of Mathematics, Graduate School of Science, Hiroshima University, 1-3-1 Kagamiyama, Higashi-Hiroshima 739-8526, Japan

e-mail: ichiro-shimada@hiroshima-u.ac.jp 